\title{
Novedades en la distribución geográfica del grupo verrucarum (Diptera: Psychodidae) en Colombia
}

\author{
Eduar Elías Bejarano, Diana Sierra, Iván Darío Vélez \\ Programa de Estudio y Control de Enfermedades Tropicales (PECET), \\ Universidad de Antioquia, Medellín, Colombia.
}

La incriminación de especies de Lutzomyia del grupo verrucarum Theodor, 1965, en la transmisión de leishmaniosis en Colombia ha puesto de manifiesto la necesidad de conocer en detalle su distribución geográfica. Este trabajo pretende contribuir a su conocimiento mediante la incorporación de 34 registros nuevos, entre los que sobresalen el hallazgo de Lutzomyia spinicrassa en un bosque seco tropical de la Costa Atlántica, la presencia de Lutzomyia ovallesi en la Orinoquia y Amazonia y la simpatría de L. spinicrassa y Lutzomyia quasitownsendi en la Cordillera Oriental. Complementariamente, este artículo recopila y sintetiza la distribución municipal y altitudinal de las 19 especies registradas en el país. Los datos compilados muestran que la serie townsendi (L. spinicrassa, Lutzomyia longiflocosa, L. quasitownsendi, Lutzomyia sauroida, Lutzomyia torvida, Lutzomyia townsendi, Lutzomyia youngi) está generalmente asociada con los pisos altitudinales premontano y montano bajo, mientras la serie verrucarum hace presencia desde el piso tropical hasta el montano con taxones de extenso ámbito geográfico (Lutzomyia evansi, Lutzomyia columbiana, $L$. ovallesi, Lutzomyia nuneztovari, Lutzomyia nevesi) y taxones de distribución endémica (Lutzomyia andina, Lutzomyia disiuncta, Lutzomyia moralesi, Lutzomyia antioquiensis). De otro lado, los representantes de la serie pia (Lutzomyia pia, Lutzomyia limafalcaoae) y de la serie serrana (Lutzomyia serrana) aparecen desde el piso tropical hasta el montano bajo. Se postula que las divergencias en los rangos altitudinales podrían estar relacionadas intrínsecamente con el origen de las especies, derivándose de fenómenos climáticos y geológicos que han afectado la flora y fauna de la región andina.

Palabras clave: Psychodidae, flebotomíneos, Lutzomyia, distribución, leishmaniosis, Colombia.

New distributional records of the verrucarum species group (Diptera: Psychodidae) in Colombia

The incrimination of sand flies belonging to verrucarum species group in the leishmaniasis transmission underscores the need for a detailed information on the geographical distribution of these species. The current listing adds 34 new records that extend significantly the knowledge of the geographical distribution of the verrucarum group in Colombia. The most important new records pertain to Lutzomyia spinicrassa in the tropical dry forest of the Atlantic coast, Lutzomyia ovallesi in the Orinoco and Amazon River watersheds regions of Colombia, and the sympatric occurrence of Lutzomyia spinicrassa and Lutzomyia quasitownsendi in the eastern chain of the Andes mountains. Additionally, the municipal and altitudinal distributions are compiled for 19 new species recorded for Colombia. The series townsendi of the verrucarum group generally restricted to premontane and lower montane zones based on distribution data on Lutzomyia longiflocosa, L. quasitownsendi, Lutzomyia sauroida, $L$. spinicrassa, Lutzomyia torvida, Lutzomyia townsendi and Lutzomyia youngi. The series verrucarum is distributed from the tropical lowland to the montane zones, and includes species with wide geographical range (Lutzomyia evansi, Lutzomyia columbiana, L. ovallesi, Lutzomyia nuneztovari, Lutzomyia nevesi), and species of highly endemic distribution (Lutzomyia andina, Lutzomyia disiuncta, Lutzomyia moralesi, Lutzomyia antioquiensis). Members of the series pia (Lutzomyia pia, Lutzomyia limafalcaoae) and the series serrana 
(Lutzomyia serrana) occur from the tropical lowlands to the lower montane zones. The altitudinal divergences may be intrinsically tied to speciation process, especially as it relates to the climatic and geologic events that have affected the flora and fauna of the Andean region.

Key words: Psychodidae, sand flies, Lutzomyia, distribution, leishmaniasis, Colombia.

El conocimiento de la distribución geográfica de los insectos vectores es básico para el control de las enfermedades que transmiten, entre ellas la leishmaniosis, llevada al humano por nematóceros de los géneros Lutzomyia França, 1924, y Phlebotomus Rondani \& Berté, 1840 (1). Además de su importancia epidemiológica, esta información constituye el fundamento de los estudios biogeográficos por métodos analíticos (2). Entre las especies de Lutzomyia del grupo verrucarum Theodor, 1965, presentes en Colombia, se encuentran reconocidos vectores de leishmaniosis $(3,4)$, lo que explica que gran parte de las investigaciones médico-entomológicas nacionales giren en torno al taxón.

La primera mención del grupo en el país correspondió a Ristorcelli y Van Ty (5), que describieron a L. columbiana (Ristorcelly y Van Ty, 1941) y registraron L. evansi (Nuñez-Tovar, 1924), seguida a su vez del hallazgo de $L$. serrana (Damasceno y Arouck, 1949) por Sherlock (6). Entre 1967 y 1974, Osorno-Mesa y colaboradores (7-13) realizaron destacadas contribuciones representadas por la descripción de seis nuevos taxones (L. spinicrassa Morales, Osorno, Osorno y Muñoz, 1969, L. longiflocosa Osorno, Morales, Osorno y Muñoz, 1970, L. andina Osorno, Osorno y Morales, 1972, L. quasitownsendi Osorno, Osorno y Morales, 1972, L. sauroida Osorno, Morales y Osorno, 1972, L. disiuncta Morales, Osorno y Osorno, 1974), la detección de L. ovallesi (Ortiz, 1952) y L. pia (Fairchild y Hertig, 1961) y la recopilación de la distribución geográfica del grupo. Posteriormente, Young amplió el número de especies conocidas con la descripción de Lutzomyia moralesiYoung, 1979 (14), y el registro

\footnotetext{
Correspondencia:

Eduar E. Bejarano, PECET, Universidad de Antioquia, Carrera 50 A No. 63-85, Medellín, Colombia. Apartado aéreo 1226. Teléfono: 263 5555; Fax: 5162675.

bejarano@medicina.udea.edu.co
}

Recibido: 04/03/03; aceptado: 04/07/03 de L. nuneztovari (Ortiz, 1954) y L. nevesi (Damasceno y Arouck, 1956) (15), gracias a un exhaustivo estudio de la fauna flebotomínea colombiana.

A principios de los noventa, Vélez y colaboradores (16) y Warburg y colaboradores (17) informaron la presencia de L. youngi y L. townsendi, respectivamente. Este hallazgo fue seguido por la descripción de Lutzomyia torvida Young, Morales y Ferro, 1994 (18). En 1999, MontoyaLerma y Ferro (19) presentaron la distribución departamental de 16 especies del grupo, la cual apareció el mismo año en la página web del Computer-aided identification of phlebotomine sand flies of Americas, CIPA (20). Finalmente, con la reciente descripción de $L$. limafalcaoae (Wolff y Galati, 2002) y L. antioquiensis (Wolff y Galati, 2002) (21), se elevan a 19 los taxones registrados en el país (22).

El objetivo primario de este artículo fue aportar nuevos registros al grupo verrucarum en Colombia. En segundo lugar, se intentó recopilar la distribución municipal y el rango altitudinal de cada uno de los taxa, dada la necesidad de que las autoridades de salud locales dispongan de información detallada respecto de la repartición de las especies vectoras. Por último, se retomaron algunos aspectos básicos concernientes a la biogeografía e importancia médica del grupo en el territorio nacional.

\section{Materiales y métodos}

Los nuevos registros de campo del grupo verrucarum fueron obtenidos por investigadores y colaboradores del Programa de Estudio y Control de Enfermedades Tropicales, Pecet, de la Universidad de Antioquia, durante estudios ecoepidemiológicos realizados en focos de leishmaniosis. Los especímenes de los cuales se derivaron estos registros se recolectaron con capturadores bucales (en reposo e intentando picar al humano), trampas de papel impregnadas en aceite de ricino y trampas de luz tipo Shannon y 
CDC. En el cuadro 1 se detalla el método de captura utilizado por especie y localidad. Cada ejemplar fue identificado por medio de la clave taxonómica de Young y Duncan (18), utilizando también la clave dicotómica de Wolff y Galati (21) para las series verrucarum y pia. La determinación taxonómica de las especies de la serie townsendi se realizó a partir de ejemplares machos, debido al isomorfismo que caracteriza a las hembras.

Para compilar la distribución municipal y el rango altitudinal del grupo verrucarum se realizó una búsqueda exhaustiva en las publicaciones existentes hasta la fecha, repartidas en las siguientes categorías: artículos extensos y cortos, resúmenes y memorias de eventos científicos, tesis, capítulos de libros y base de datos del CIPA. Las localidades señaladas explícitamente en su respectiva fuente bibliográfica como datos no publicados no se incluyeron en la recopilación. Para cada especie y municipio se tomó la primera citación en la literatura. No obstante, si después de una tesis o resumen de un congreso, los mismos autores publicaron un artículo, se citó este último.

\section{Resultados y discusión}

Se adicionan 34 registros nuevos en la distribución del grupo verrucarum, lo que eleva aproximadamente a 133 los municipios del país donde éste hace presencia (cuadro 1) (23-67). Cada una de las series de especies (verrucarum, serrana, townsendi, pia) posee representantes en Colombia, lo que le facilita al grupo la cobertura de los pisos altitudinales tropical (0-500 metros sobre el nivel del mar (msnm)), premontano (500$1.500 \mathrm{msnm})$, montano bajo (1.500-2.500 $\mathrm{msnm})$ y montano (2.500-3.500 msnm).

La serie townsendiaparece representada por siete taxones de distribución endémica: $L$. spinicrassa, L. sauroida, L. quasitownsendi, L. torvida, L. longiflocosa, L. youngi y L. townsendi. Esta serie está limitada a los pisos premontano y montano bajo de las Cordilleras Central y Oriental, con la excepción de $L$. spinicrassa, un reconocido transmisor de Leishmania braziliensis (3), que habita desde los 218 hasta los $1.690 \mathrm{msnm}(32,48)$ alcanzando la Costa Atlántica. La presencia de $L$. spinicrassa en la zona periurbana de Sincelejo, departamento de Sucre (cuadro 1), además de su relevancia epidemiológica, amplía el rango ecológico para la especie, constituyéndose en su primer hallazgo en un bosque seco tropical.

L. sauroida, L. quasitownsendi y L. torvida ocupan la Cordillera Oriental. L. sauroida está presente entre los 850 y los $1.780 \mathrm{msnm}(11,48)$. L. quasitownsendi, encontrada en la naturaleza con flagelados no identificados (53), se extiende desde los 1.140 hasta los $1.780 \mathrm{msnm}(31,68)$. L. torvida ha sido registrada entre los 1.400 y $1.900 \mathrm{msnm}$, en dos municipios del departamento de Cundinamarca (cuadro 1) asentados sobre la vertiente occidental de la cordillera, donde se halló con flagelados no identificados (69). Adicionalmente, $L$. torvida ha mostrado estar en capacidad de soportar, experimentalmente, la infección por parásitos de $L$. braziliensis procedentes de la misma región (70).

L. longiflocosa, que también se infectó bajo condiciones de laboratorio con $L$. braziliensis y lo transmitió por picadura a un hospedero (71), aparece entre las Cordilleras Central y Oriental en pisos altitudinales que van de los 900 a los $2.100 \mathrm{msnm}$ (42). Este insecto es abundante entre los 1.500 y $1.700 \mathrm{msnm}$ y se considera como posible vector en algunas localidades $(42,58)$. $L$. youngi se presenta en la estribación occidental de la Cordillera Central, en elevaciones de 990 a $1.850 \mathrm{msnm}(16,32)$, y muestra una mayor densidad entre los 1.500 y $1.800 \mathrm{msnm}$ (16). Esta especie ha sido infectada experimentalmente en el país con L. braziliensis y Leishmania infantum $(17,72)$, tornándose en un potencial transmisor a lo largo de su distribución. L. townsendi habita sobre la Cordillera Occidental, entre los 1.450 y los 1.799 msnm (67), y actúa, probablemente, como un vector de L. braziliensis en el departamento de Valle del Cauca, habiéndose constatado su capacidad de soportar el desarrollo del agente patógeno (17).

En la serie verrucarum aparecen taxones de extenso ámbito geográfico (L. evansi, L. columbiana, $L$. ovallesi, $L$. nuneztovari, $L$. nevesi) y taxones confinados (L. andina, L. disiuncta, L. moralesi, $L$. antioquiensis). L. evansi es una de las especies del grupo verrucarum con mayor cobertura en 
Cuadro 1. Distribución geográfica de las especies de Lutzomya del grupo verrucaum Theodor, 1965, en Colombia.

\begin{tabular}{|c|c|c|}
\hline Departamento & Especie & Municipio o localidad (referencias) \\
\hline Amazonas & $\begin{array}{l}\text { Lutzomyia nevesi } \\
\text { L. ovallesi }\end{array}$ & $\begin{array}{l}\text { Leticia }(15) \\
\text { Tarapacá }\left({ }^{*}\right)^{a}\end{array}$ \\
\hline Antioquia & $\begin{array}{l}\text { L. antioquiensis } \\
\text { L. columbiana } \\
\text { L. evansi } \\
\text { L. limafalcaoae } \\
\text { L. moralesi } \\
\text { L. nuneztovari } \\
\text { L. ovallesi } \\
\text { L. pia } \\
\text { L. serrana } \\
\text { L. youngi }\end{array}$ & $\begin{array}{l}\text { Montebello }(21) \\
\text { Envigado }(23) \text {, Gómez Plata }\left({ }^{*}\right)^{c} \text {, Medellín }(24) \\
\text { San Roque }(25) \\
\text { Montebello }(21) \\
\text { Yolombó }\left(^{*}\right)^{\mathrm{a}, \mathrm{b}} \\
\text { Montebello }(16) \\
\text { Carepa }\left({ }^{*}\right)^{\mathrm{a}} \text {, Chigorodó }(13) \text {, San Luis }(26) \text {, San Roque (27) } \\
\text { Envigado }(23) \text {, Ituango }\left({ }^{*}\right)^{\mathrm{a}} \text {, San Francisco }\left({ }^{*}\right)^{\mathrm{b}} \\
\text { Anorí (14) } \\
\text { Montebello (16), Santa Bárbara (27) }\end{array}$ \\
\hline Bolívar & $\begin{array}{l}\text { L. evansi } \\
\text { L. serrana }\end{array}$ & $\begin{array}{l}\text { Arjona (14), Carmen de Bolívar (28), Isla Fuerte (29), Mompós (13), } \\
\text { Santa Rosa de Lima }\left({ }^{*}\right)^{a, c, d} \\
\text { Simití (13) }\end{array}$ \\
\hline Boyacá & $\begin{array}{l}\text { L. nuneztovari } \\
\text { L. ovallesi } \\
\text { L. pia } \\
\text { L. quasitownsendi } \\
\text { L. sauroida } \\
\text { L. serrana } \\
\text { L. spinicrassa }\end{array}$ & $\begin{array}{l}\text { Otanche (30) } \\
\text { Otanche (30), Puerto Boyacá (13) } \\
\text { Almeida (13), Otanche (30) } \\
\text { Santana (31) } \\
\text { Santana (11) } \\
\text { Otanche (30), Puerto Boyacá (13) } \\
\text { Almeida (8), Guateque (8), Guayatá (8) }\end{array}$ \\
\hline Caldas & $\begin{array}{l}\text { L. columbiana } \\
\text { L. nuneztovari } \\
\text { L. ovallesi } \\
\text { L. pia } \\
\text { L. serrana } \\
\text { L. youngi }\end{array}$ & $\begin{array}{l}\text { Aguadas }\left({ }^{*}\right)^{\mathrm{a}} \text {, Filadelfia }(32) \text {, Pensilvania (32) } \\
\text { Salamina }(32) \\
\text { Aguadas }(32) \text {, Victoria }(13) \\
\text { Filadelfia }(32) \text {, Salamina (32) } \\
\text { Samaná }\left({ }^{*}\right)^{\mathrm{e}} \text {, Victoria (13) } \\
\text { Aguadas }(32) \text {, Filadelfia (32), Salamina (32) }\end{array}$ \\
\hline Caquetá & L. evansi & $(20)^{+}$ \\
\hline Casanare & $\begin{array}{l}\text { L. evansi } \\
\text { L. ovallesi }\end{array}$ & $\begin{array}{l}\text { Hato Corozal (25), Paz de Ariporo (25), Tauramena (25) } \\
\text { Monterrey }\left({ }^{*}\right)^{\mathrm{e}}\end{array}$ \\
\hline Cauca & L. columbiana & Bolívar (6), Popayán (33) \\
\hline Cesar & L. disiuncta & Agustín Codazzi (12) \\
\hline Chocó & $\begin{array}{l}\text { L. ovallesi } \\
\text { L. serrana }\end{array}$ & $\begin{array}{l}\text { Bahía Solano }\left({ }^{*}{ }^{a} \text {, Riosucio (14) }\right. \\
\text { Juradó }(14)\end{array}$ \\
\hline Córdoba & $\begin{array}{l}\text { L. evansi } \\
\text { L. serrana }\end{array}$ & $\begin{array}{l}\text { Cereté (25), Chinú (28), Sahagún (34), San Andrés de Sotavento (35) } \\
\text { San Andrés de Sotavento (36) }\end{array}$ \\
\hline Cundinamarca & $\begin{array}{l}\text { L. andina } \\
\text { L. disiuncta } \\
\text { L. evansi } \\
\text { L. nuneztovari } \\
\text { L. ovallesi } \\
\\
\text { L. pia } \\
\text { L. serrana } \\
\text { L. torvida }\end{array}$ & 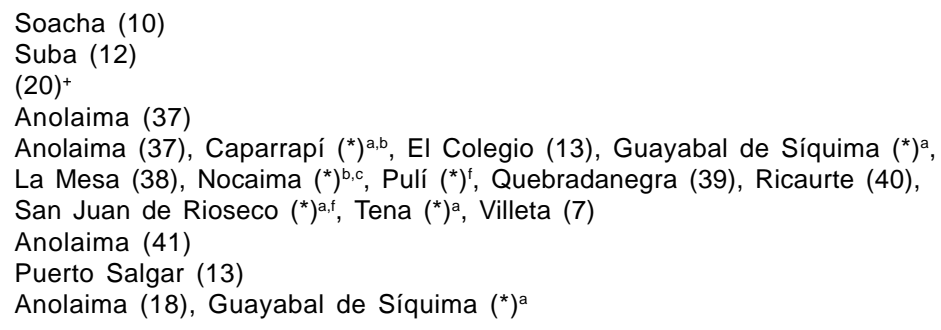 \\
\hline Huila & $\begin{array}{l}\text { L. columbiana } \\
\text { L. longiflocosa } \\
\text { L. nuneztovari } \\
\text { L. pia }\end{array}$ & $\begin{array}{l}\text { Baraya (37), Tello (37) } \\
\text { Algeciras (42), Baraya (37), Garzón (42), Neiva (42), Santa María (42), } \\
\text { Tello (9) } \\
\text { Algeciras (42), Baraya (37), Garzón (42), lquira (42), Neiva (42), } \\
\text { Saladoblanco (42), Santa María (42), Tello (37), Timaná (14) } \\
(19)^{+}\end{array}$ \\
\hline La Guajira & $\begin{array}{l}\text { L. columbiana } \\
\text { L. evansi }\end{array}$ & $\begin{array}{l}\text { Maicao (13), Uribia (13) } \\
\text { Maicao (13), Uribia (13), Urumita (13) }\end{array}$ \\
\hline
\end{tabular}




\begin{tabular}{|c|c|c|}
\hline Departamento & Especie & Municipio o localidad (referencias) \\
\hline Magdalena & $\begin{array}{l}\text { L. evansi } \\
\text { L. ovallesi }\end{array}$ & $\begin{array}{l}\text { Santa Marta (14) } \\
\text { Santa Marta (14) }\end{array}$ \\
\hline Meta & $\begin{array}{l}\text { L. evansi } \\
\text { L. nevesi } \\
\text { L. pia } \\
\text { L. Serrana }\end{array}$ & $\begin{array}{l}\text { La Macarena (43) } \\
\text { San Juan de Arama (20) } \\
\text { La Macarena (43) } \\
(20)^{+}\end{array}$ \\
\hline Nariño & $\begin{array}{l}\text { L. evansi } \\
\text { L. pia } \\
\text { L. serrana }\end{array}$ & $\begin{array}{l}\text { Ancuyá (13), Colón (13), Consacá (13), Imués (13), La Unión (13), } \\
\text { Samaniego (13), San Pablo (6), Sandoná (13), Túquerres (6), Valle del } \\
\text { Capulí (5) } \\
\text { Valle del Capulí (5) } \\
\text { Tumaco (44) } \\
\text { Tumaco (45) }\end{array}$ \\
\hline Norte de Santander & $\begin{array}{l}\text { L. evansi } \\
\text { L. nuneztovari } \\
\text { L. ovallesi } \\
\text { L. pia } \\
\text { L. quasitownsendi } \\
\text { L. sauroida } \\
\text { L. serrana } \\
\text { L. spinicrassa }\end{array}$ & 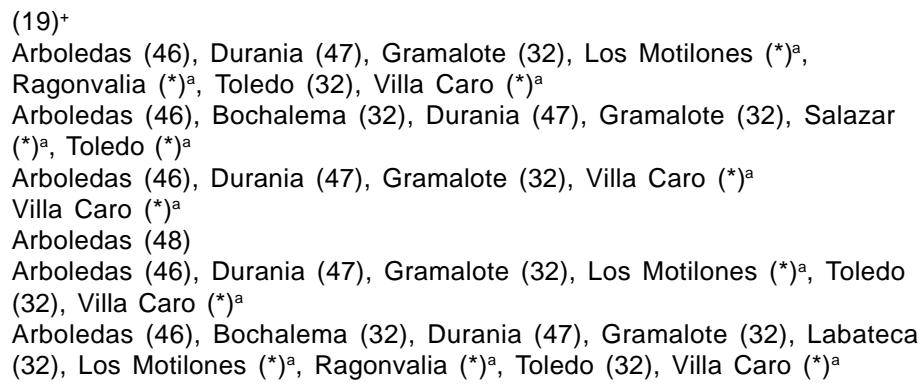 \\
\hline Putumayo & L. nevesi & Puerto Asís (49) \\
\hline Risaralda & $\begin{array}{l}\text { L. columbiana } \\
\text { L. pia }\end{array}$ & $\begin{array}{l}\text { Guática (33), Marsella (32) } \\
\text { Guática (33), Marsella (32), Pereira (33) }\end{array}$ \\
\hline Santander & $\begin{array}{l}\text { L. evansi } \\
\text { L. ovallesi } \\
\text { L. quasitownsendi } \\
\text { L. serrana }\end{array}$ & $\begin{array}{l}\text { San Vicente de Chucurí (50) } \\
\text { Bucaramanga (51), Piedecuesta (52), Socorro (13) } \\
\text { Barbosa (10), Bucaramanga (51), Güepsa (10), Landázuri (53), } \\
\text { Socorro (10) } \\
\text { San Vicente de Chucurí (6) }\end{array}$ \\
\hline Sucre & $\begin{array}{l}\text { L. evansi } \\
\text { L. ovallesi } \\
\text { L. serrana } \\
\text { L. spinicrassa }\end{array}$ & $\begin{array}{l}\text { Chalán (34), Colosó (34), Coveñas (54), Galeras (34), Los Palmitos (34), } \\
\text { Morroa (34), Ovejas (34), Sampués (55), San Juán de Betulia (34), San } \\
\text { Pedro (34), Sincelejo (56), Tolú (34), Toluviejo (34) } \\
(19)^{+} \\
\text {Colosó }(57) \text {, Sampués (55) } \\
\text { Sincelejo }\left(^{*}\right)^{\text {a }}\end{array}$ \\
\hline Tolima & $\begin{array}{l}\text { L. Columbiana } \\
\text { L. longiflocosa } \\
\text { L. nuneztovari } \\
\text { L. ovallesi } \\
\text { L. pia } \\
\text { L. serrana }\end{array}$ & $\begin{array}{l}\text { Planadas (58), Rovira (18) } \\
\text { Casablanca (42), Herveo (59), Planadas }(58) \text {, Ortega }\left({ }^{*}\right)^{\mathrm{a}} \text {, Rovira }(18) \\
\text { Planadas }(58) \\
\text { Herveo }\left(^{*}\right)^{\mathrm{a}} \text {, Mariquita }(60) \\
\text { Herveo }\left(^{*}\right)^{\mathrm{a}} \\
\text { Mariquita }(60)\end{array}$ \\
\hline Valle del Cauca & $\begin{array}{l}\text { L. columbiana } \\
\text { L. evansi } \\
\text { L. moralesi } \\
\text { L. pia } \\
\text { L. serrana } \\
\text { L. townsendi } \\
\text { L. youngi }\end{array}$ & $\begin{array}{l}\text { Alto Aguacatal (61), Dagua (62), El Carmen (14), La Cumbre (62), } \\
\text { Restrepo (63), Tuluá (17), Ulloa (64), Versalles (17), Yotoco (62) } \\
\text { Versalles (17) } \\
\text { Pichindé (14), Rio Pance (14) } \\
\text { Versalles (17), Yotoco (17) } \\
\text { Tuluá (65), Ulloa (64) } \\
\text { Dagua (66), La Cumbre (67), Restrepo (63), Versalles (17) } \\
\text { Tuluá (20) }\end{array}$ \\
\hline
\end{tabular}

* Registro nuevo

+ Localidad no especificada en la literatura

Método de captura utilizado para recolectar los flebotomíneos que constituyen nuevos registros: aShannon,

${ }^{b}$ cebo humano, ${ }^{c} \mathrm{CDC}$, dCebo animal, etrampa de papel, 'reposo 
Colombia, 14 departamentos (cuadro 1), incluso una población marítimamente aislada, Isla Fuerte (29), originada presumiblemente por dispersión desde la Costa Atlántica (25). Esta especie, que ha sido comprobada como vector principal de $L$. infantum en el norte del país (4), abarca desde los 4 hasta los $1.450 \mathrm{msnm}$ (25). A pesar de su amplio rango geográfico, $L$. evansi presenta restricciones altitudinales con respecto a su abundancia, con preferencia por los bosques secos tropicales (0-300 msnm), no obstante su presencia en ambientes húmedos $(17,25)$.

L. ovallesi también cubre gran parte del territorio colombiano, 12 departamentos (cuadro 1), en un margen de distribución que va desde los 3 hasta los $2.160 \mathrm{msnm}(14,32)$, con una mayor densidad en el piso premontano, entre los 800 y 1.200 msnm. El informe de L. ovallesi en Tarapacá, departamento del Amazonas (cuadro 1), constituye el registro más meridional de la especie en Suramérica. Adicional a su hallazgo en el país con flagelados no identificados (3), también se ha verificado su capacidad de soportar el desarrollo de $L$. braziliensis (70), lo que permite señalarla como un potencial transmisor del parásito $(3,39,51,70)$. L. columbiana se extiende desde bosques húmedos del Nudo de Los Pastos, pasando por las Cordilleras Occidental y Central, hasta zonas esteparias de La Guajira, en un rango altitudinal de 100 a 2.700 msnm $(6,13)$. Este flebotomíneo ha sido infectado en el laboratorio con L. braziliensis (17) y Leishmania mexicana (73), y se ha postulado como transmisor en el suroccidente del país $(17,66,73-75)$.

L. nuneztovari muestra un patrón de distribución caracterizado por su baja abundancia y común acompañamiento de otros taxones del grupo como L. ovallesi, L. spinicrassa, L. torvida, L. youngi, $L$. longiflocosa, L. columbiana, L. sauroida, $L$. serrana y $L$. pia $(32,37,47,48,58)$, en alturas que oscilan entre los 850 y los 2.100 msnm $(42,48)$. En Colombia, L. nuneztovari ha mostrado ser susceptible a la infección con $L$. braziliensis bajo condiciones experimentales (70) por lo que es tratada como un probable vector secundario, dado que su baja densidad tornaría inviable el mantenimiento del ciclo de transmisión en ausencia de otros vectores (70). L. nevesiaparece en bosques húmedos tropicales y premontanos de los departamentos del Meta, Putumayo y Amazonas, en alturas de 100 a 650 msnm $(15,20,49)$.

Los restantes cuatro taxones de la serie verrucarum tienen una distribución geográfica muy limitada. L. andina es endémica de la Cordillera Oriental, aparece a $2.550 \mathrm{msnm}$ (10). L. disiuncta también está confinada a la Cordillera Oriental y sus estribaciones, pero en altitudes que contrastan entre los 132 y $2.583 \mathrm{msnm}$ (12). L. antioquiensis es propia de la Cordillera Central, se registra en cavernas ubicadas a $2.060 \mathrm{msnm}$ (21). L. moralesi ocupa las Cordilleras Central y Occidental, en un rango de 1.600 a 1.990 msnm (14); este último límite corresponde a un nuevo registro en Yolombó, departamento de Antioquia (cuadro1).

Aunque hasta la fecha se han descrito diez especies de la serie serrana (22), algunas de ellas con aparición en Centro y Suramérica, en Colombia sólo se ha encontrado a $L$. serrana. Sin embargo, es posible que otros taxones estén presentes, dado que por su ubicación geográfica el país pudo ser un paso obligado en la dispersión de dicha serie. L. serrana cubre 14 departamentos (cuadro 1), en un intervalo de 20 a $1.625 \mathrm{msnm}(32,45)$, presenta una mayor densidad entre los 700 y 1.200 msnm (18). Su ámbito biogeográfico abarca desde las costas Atlántica $(36,57)$ y Pacífica $(14,45)$ hasta zonas montañosas de las distintas cordilleras andinas $(13,30,32,64)$. A pesar de exhibir cierto comportamiento antropofílico a lo largo de esta distribución $(3,47,48)$, no existen evidencias del papel vectorial de $L$. serrana en Colombia.

La serie pia está representada por dos especies, L. pia y L. limafalcaoae. La primera se extiende por las Cordilleras Oriental, Central y Occidental, en un rango altitudinal de 100 a 1.925 msnm $(13,44)$, mientras la segunda es propia de la Cordillera Central, y se encuentra a 2.060 msnm (21). L. pia se distribuye simpátricamente con diferentes especies del grupo verrucarum en zonas cafeteras $(17,32,46-48)$, lo que podría ser una constancia de su origen evolutivo común (22). Además de picar al humano $(23,46-48)$, L. pia ha sido infectada experimentalmente con $L$. braziliensis (17). 
En el grupo verrucarum se aprecian patrones divergentes de repartición geográfica, especialmente entre las series townsendi y verrucarum, e incluso dentro de esta última. Estas diferencias podrían estar relacionadas intrínsecamente con el origen de las especies, en tanto que cada una de éstas como unidad biológica puede responder de manera distinta a las mismas presiones climáticas o geológicas, lo que determina su permanencia o desaparición en una zona geográfica. En este sentido, se ha propuesto que la actual distribución del grupo es el resultado de una larga historia climática y geológica, en la que se destacan los episodios paleoclimáticos del Cuaternario, el elevamiento de los Andes y el surgimiento del istmo de Panamá como los eventos de mayor relevancia, de los cuales se deriva a su vez la extraordinaria riqueza de especies del noroccidente sudamericano (22).

Es notorio que la distribución que exhiben los taxones parece confirmar la existencia de menos especies en áreas secas, tal como se ha demostrado en el país para especies vegetales (76). Mientras en bosques secos de la Costa Atlántica se ha descrito la presencia de máximo dos miembros del grupo verrucarum $(13,36,57)$, en bosques húmedos del interior del país se registra la yuxtaposición de hasta seis de éstos (48), algunos de ellos isomórficos, lo que tiene trascendencia epidemiológica. La simpatría de $L$. spinicrassa y $L$. quasitownsendi en el foco de leishmaniosis de Villa Caro, Norte de Santander (cuadro1), dificulta la incriminación de la especie vectora en el área, lo cual hace necesario recurrir a herramientas taxonómicas alternas que permitan discriminar las hembras (77). Este hallazgo se suma al previo registro simpátrico de $L$. spinicrassa y $L$. sauroida en otra localidad de este departamento (48).

Las implicaciones evolutivas de estas simpatrías podrían llegar a inferirse, en la medida en que son el producto de procesos asociados con el origen de los taxones. De este modo, si las especies se derivaron recientemente y sus áreas de distribución se superponen, su diversificación estará en función del desarrollo de mecanismos de aislamiento reproductivo y de competencia interespecífica entre ellas. Por tanto, la coexistencia espacial de componentes isomórficos del complejo townsendi, aunque puede constituir per se una prueba ecológica de su estado específico, merece un profundo análisis biogeográfico y filogenético, cuyos resultados contribuirían al conocimiento de la génesis de la serie.

\section{Agradecimientos}

Los autores expresan sus agradecimientos a los funcionarios de los Servicios de Salud departamentales que colaboraron en la recolección de especímenes en el campo, especialmente a Eulides Pabón. Este artículo se deriva en parte de un proyecto de investigación financiado por el Instituto Colombiano para el Desarrollo de la Ciencia y la Tecnología Francisco José de Caldas, Colciencias (115-05-106-97). El autor principal recibió apoyo financiero del Programa de Formación de Jóvenes Investigadores de Colciencias.

\section{Referencias}

1. Killick-Kendrick R. The biology and control of phlebotomine sand flies. Clin Dermatol 1999;17:279-89.

2. Williams P. Patterns in the geographical distribution of members of the genus Lutzomyia França (Diptera: Psychodidae - Phlebotominae). En: Burger JF, editor. Contributions to the knowledge of Diptera. Gainesville: Associated Publishers; 1999. p.455-502.

3. Young DG, Morales A, Kreutzer RD, Alexander JB, Corredor A, Tesh RB, et al. Isolations of Leishmania braziliensis (Kinetoplastida: Trypanosomatidae) from cryopreserved Colombian sand flies (Diptera: Psychodidae). J Med Entomol 1987;24:587-9.

4. Travi BL, Vélez ID, Brutus L, Segura I, Jaramillo C, Montoya J. Lutzomyia evansi, an alternate vector of Leishmania chagasi in a Colombian focus of visceral leishmaniasis. Trans R Soc Trop Med Hyg 1990; 84:676-7.

5. Ristorcelli A, Van Ty D. III. Phlébotomes d'une région de Colombie où la verruga du Pérou est devenue endémique depuis deux ou trois ans (Seconde note). Ann Parasitol Hum Comp 1941;18:251-69.

6. Sherlock IA. Sôbre alguns Phlebotomus e Brumptomyia da Colômbia (Diptera, Psychodidae). Mem Inst Oswaldo Cruz 1962;60:321-36.

7. Osorno-Mesa E, Morales-Alarcón A, Osorno F. Phlebotominae de Colombia (Diptera: Psychodidae) I. Distribución geográfica de especies de Phlebotomus registradas con algunas anotaciones biológicas y descripción de una nueva. Caldasia 1967;10:27-34. 
8. Morales-Alarcón A, Osorno-Mesa E, De Osorno F, Muñoz de Hoyos P. Phlebotominae de Colombia (Diptera, Psychodidae). V. Descripción de una nueva especie de Lutzomyia. Rev Acad Colomb Cienc 1969; 13:383-90.

9. Osorno-Mesa E, Morales-Alarcón A, Osorno F, Muñoz de Hoyos P. Phlebotominae de Colombia (Diptera, Psychodidae). VI. Descripción de Lutzomyia longiflocosa n. sp. y Lutzomyia bifoliata $\mathrm{n}$. sp. Bol Mus Nat Hist Univ Fed Minas Gerais 1970;6:1-18.

10. Osorno F, Osorno-Mesa E, Morales-Alarcón A. Phlebotominae de Colombia (Diptera: Psychodidae). VII. Descripción de Lutzomyia andina n. sp. y Lutzomyia quasitownsendin. sp. Bol Mus Nat Hist Univ Fed Minais Gerais 1972;12:1-29.

11. Osorno-Mesa E, Morales-Alarcón A, Osorno F. Phlebotominae de Colombia. VIII. Descripción de Lutzomyia sauroida sp.n. y Lutzomyia caprina sp.n. (Diptera, Psychodidae). Rev Bras Biol 1972;32:433-41.

12. Morales-Alarcón A, Osorno F, Osorno-Mesa E. Phlebotominae de Colombia. X. Descripción de la hembra de Lutzomyia dendrophila (Mangabeira, 1942) y del macho y la hembra de Lutzomyia disiuncta sp. $\mathrm{n}$. (Diptera, Psychodidae). Rev Bras Biol 1974;34:443-52.

13. Osorno-Mesa E, Morales-Alarcón A, Osorno F, Ferro-Vela C. Phlebotominae de Colombia (Diptera, Psychodidae). IX. Distribución geográfica de especies de Brumptomyia França y Parrot, 1921 y Lutzomyia França, 1924 encontradas en Colombia, S.A. Rev Acad Colomb Cienc 1972;14:45-68.

14. Young DG. A review of the bloodsucking psychodid flies of Colombia (Diptera: Phlebotominae and Sycoracinae). Technical Bulletin 806, Gainesville, Florida: Agricultural Experiment Stations, Institute of Food and Agricultural Sciences; 1979. p.1-265.

15. Young DG, Morales A. New species and records of phlebotomine sand flies from Colombia (Diptera: Psychodidae). J Med Entomol 1987;24:651-65.

16. Vélez ID, Wolff M, Valderrama R, Escobar JP, Osorio L. Community and environmental risk factors associated with cutaneous leishmaniasis in Montebello, Antioquia, Colombia. En: Wijeyaratne P, Goodman T, Espinal C, editores. Leishmaniasis control strategies. A Critical evaluation of IDRC-supported research. Ottawa: Editorial International Development Research Center; 1991. p.261-74.

17. Warburg A, Montoya-Lerma J, Jaramillo C, CruzRuiz AL, Ostrovska K. Leishmaniasis vector potential of Lutzomyia spp. in Colombian coffee plantations. Med Vet Entomol 1991;5:9-16.

18. Young DG, Duncan MA. Guide to the identification and geographic distribution of Lutzomyia sand flies in Mexico, the West Indies, Central and South America (Diptera: Psychodidae). Mem Amer Ent Inst 1994;54:1881.
19. Montoya-Lerma J, Ferro C. Flebótomos (Diptera: Psychodidae) de Colombia. En: Amat G, Andrade MG, Fernández $\mathrm{F}$, editores. Insectos de Colombia. Volumen II. Colección Jorge Alvarez Lleras, No. 13. Academia Colombiana de Ciencias Exactas, Físicas y Naturales. Santafé de Bogotá: Centro Editorial Javeriano; 1999. p.211-45.

20. Balard Y, Bermudez H, Dedet JP, Falcao AL, Feliciangeli MD, Ferro C, et al. Computer-aided identification of phlebotomine sand flies of America, 1999. Disponible en URL: http://cipa.snv.jussieu.fr/

21. Wolff M, Galati EAB. Description of Pintomyia limafalcaoae and Pintomyia antioquiensis, two new species of phlebotomine sand fly (Diptera, Psychodidae) from the Colombian Andes. Mem Inst Oswaldo Cruz 2002;97:317-24

22. Bejarano EE, Rojas W, Uribe S, Vélez ID. Sistemática de especies de Lutzomyia del grupo verrucarum Theodor, 1965 (Diptera: Psychodidae). Biomédica 2003; 23:87-102.

23. Agudelo LA, Uribe J, Sierra D, Ruiz F, Vélez ID. Presence of American cutaneous leishmaniasis vectors surrounding the city of Medellín, Colombia. Mem Inst Oswaldo Cruz 2002;97:641-2.

24. Zuleta BRA. Determinación de la fauna flebotomínea del área periurbana del Valle de Aburrá (tesis). Medellín, Colombia: Universidad de Antioquia; 2002.

25. Bejarano EE. Variabilidad genética y especiación en Lutzomyia (verrucarum) evansi (Nuñez-Tovar, 1924), vector de leishmaniosis visceral americana (tesis). Medellín, Colombia: Universidad de Antioquia; 2001.

26. López Y, Osorio L, Alvarez G, Rojas J, Jiménez F, Gómez ME, et al. Foco de leishmaniasis Río Claro, San Luis, 1991. Bol Epidemiol de Antioquia 1992;17:5969.

27. Wolff MI. Estratificación de flebotomíneos en focos de leishmaniosis en el departamento de Antioquia. Memorias, Seminario taller nacional: leishmaniosis: manejo clínico, epidemiológico y del vector. Medellín, Colombia: Servicio Seccional de Salud de Antioquia; 1993. p.139-60.

28. Vélez ID, Gallego JI, Adarve JC, Llano R, Trujillo GA, Alzate AM. Ecoepidemiological delimitation of visceral leishmaniasis in the Caribbean Colombian coast. Bol Dir Malariol y San Amb 1995;35:359-70.

29. Gallego JI, Vélez ID. Presencia en Isla Fuerte, Bolívar, de Lutzomyia evansi, vector de leishmaniosis visceral. latreia 1994;7:33-5.

30. Molina JA. Determinación de la fauna flebotomínea en el noroccidente de Boyacá, Colombia. Implicación de Lutzomyia trapidoi como especie vectora en el foco de leishmaniosis cutánea de La Zambera (tesis). Santafé de Bogotá, Colombia: Universidad de Los Andes; 1995.

31. Cabrera OL, Ferro C. Ciclo de vida de Lutzomyia spinicrassa, L. quasitownsendi y L. youngi, especies 
del grupo verrucarum (Diptera: Psychodidae). Actual Biol 2000;22:225-32.

32. Alexander B, Agudelo LA, Navarro F, Ruiz F, Molina J, Aguilera G, et al. Phlebotomine sandflies and leishmaniasis risks in Colombian coffee plantations under two systems of cultivation. Med Vet Entomol 2001; 15:364-73.

33. Barreto M, Burbano ME, Barreto P. Nuevos registros de flebotominos (Diptera: Psychodidae) y triatominos (Hemiptera: Reduviidae) para Risaralda, Cauca y Valle del Cauca, Colombia. Colombia Med 1997;28:116-22.

34. Le Pape P. Écoépidémiologie de la leishmaniose a Leishmania infantum $=$ L. chagasi dans la plaine des caraïbes (Colombie): corrélation vecteur et réservoir canin (tesis). Montpellier, Francia: Université de Montpellier I; 1992.

35. Vélez ID, Ghysais G, Marulanda J, Maya DA, Rivera I, Guerrero MA, et al. Leishmaniasis tegumentaria americana: encuesta epidemiológica en una comunidad indígena. latreia 1988;1:29-33.

36. Vélez ID, Travi BL, Gallego J, Palma GI, Agudelo $\mathrm{SP}$, Montoya J, et al. Evaluación ecoepidemiológica de la leishmaniosis visceral en la comunidad indígena zenú de San Andrés de Sotavento, Córdoba: primer paso para su control. Rev Colomb Entomol 1995;21: 111-22.

37. Ferro C, Cabrera OL, Ayala M, Santamaría E, Castillo M, Neira M. Especies del grupo verrucarum (Diptera: Psychodidae) y su papel como vectores en dos focos de Leishmania braziliensis del centro de Colombia. Resúmenes, XXV Congreso de la Sociedad Colombiana de Entomología, Cali, Colombia; 1998. p.41.

38. Barreto P. Artrópodos hematófagos del río Raposo, Valle, Colombia. IV - Psychodidae. Caldasia 1969;10: 459-72.

39. Pardo RH, Farieta S, Munstermann LE, Ferro C. Estudio preliminar de los flebótomos de Villeta y Quebradanegra, Cundinamarca: sus implicaciones en salud pública. Biomédica 1996;16:293-302.

40. Ferro C, Morrison AC, Torres M, Pardo R, Wilson ML, Tesh RB. Species composition and relative abundance of sand flies of the genus Lutzomyia (Diptera: Psychodidae) at an endemic focus of visceral leishmaniasis in Colombia. J Med Entomol 1995;32:52737.

41. Cabrera OL, Neira M, Bello F, Ferro C. Ciclo de vida y colonización de Lutzomyia ovallesi (Diptera: Psychodidae), vector de Leishmania spp. en América Latina. Biomédica 1999;19:223-9.

42. Pardo R, Ferro C, Lozano G, Lozano C, Cabrera O, Davies C. Flebótomos (Diptera: Psychodidae) vectores de leishmaniasis cutánea y sus determinantes ecológicos en la zona cafetera del Departamento del Huila. Memorias, XXVI Congreso de la Sociedad
Colombiana de Entomología, Santa Fé de Bogotá, Colombia; 1999. p.147-63.

43. Molina J, Jaramillo M, Villegas C, Guhl F. Actualización de la distribución del género Lutzomyia en Colombia. Biomédica 1997;17:152-3.

44. Alexander B, Morales AL, Becerra J, Rojas CA. Entomological aspects of a leishmaniasis control project based on community participation in riverine communities of SW Colombia. Bol Dir Malariol y San Amb 1995;35:2940.

45. Travi BL, Montoya J, Solarte Y, Lozano L, Jaramillo C. Leishmaniasis in Colombia. I. Studies on the phlebotomine fauna associated with endemic foci in the Pacific Coast region. Am J Trop Med Hyg 1988;39:261-6.

46. Tesh RB, Boshell J, Young DG, Morales A, Corredor A, Modi GB, et al. Biology of Arboledas virus, a new phlebotomus fever serogroup virus (Bunyaviridae: Phlebovirus) isolated from sand flies in Colombia. Am J Trop Med Hyg 1986;35:1310-6.

47. Tesh RB, Boshell J, Modi GB, Morales A, Young DG, Corredor A, et al. Natural infection of humans, animals, and phlebotomine sand flies with the Alagoas serotype of vesicular stomatitis virus in Colombia. Am J Trop Med Hyg 1987;36:653-61.

48. Alexander B, Ferro C, Young DG, Morales A, Tesh RB. Ecology of phlebotomine sand flies (Diptera: Psychodidae) in a focus of Leishmania (Viannia) braziliensis in northeastern Colombia. Mem Inst Oswaldo Cruz 1992;87:387-95.

49. Barreto M, Burbano ME, Barreto P. Lutzomyia sand flies (Diptera: Psychodidae) from middle and lower Putumayo Department, Colombia, with new records to the country. Mem Inst Oswaldo Cruz 2000;95:633-9.

50. Gast-Galvis A, Rengifo S. Leishmaniasis visceral. Estudio epidemiológico del primer caso diagnosticado en Colombia. Anales Soc de Biol Bogotá 1944;1:161-8.

51. Sandoval CM, Angulo VM, Gutiérrez R, Muñoz G, Ferro C. Especies de Lutzomyia (Diptera: Psychodidae) posibles vectores de leishmaniasis en la ciudad de Bucaramanga, Santander, Colombia. Biomédica 1998; 18:161-8.

52. Sandoval CM, Martínez J, Flórez M, Serrano VH, Angulo VM. Composición y abundancia estacional de flebótomos en un foco de leishmaniosis visceral en la vereda Guatiguará (Piedecuesta, Santander, Colombia). Biomédica 2002;22:111-2.

53. Muñoz G. Incrimination of Leishmania panamensis vectors by statistics methods. Memorias, XXVI Congreso de la Sociedad Colombiana de Entomología, Santa Fé de Bogotá, Colombia; 1999. p.164-71.

54. Montoya-Lerma J. The biology of visceral leishmaniasis vectors in the San Andrés de Sotavento focus, Colombia (tesis). Londres, Inglaterra: University of London; 1996. 
55. Cochero S, Blanco P. Determinación de la fauna flebotomínea en un foco de leishmaniasis en el municipio de Sampués (Sucre). Resúmenes, XXXVI Congreso Nacional de Ciencias Biológicas, Cartagena de Indias, Colombia; 2001. p.70.

56. Bejarano EE, Uribe S, Rojas W, Vélez ID. Presence of Lutzomyia evansi, a vector of American visceral leishmaniasis, in an urban area of the Colombian Caribbean coast. Trans R Soc Trop Med Hyg 2001;95: 27-8.

57. Travi BL, Adler GH, Lozano M, Cadena H, MontoyaLerma J. Impact of habitat degradation on phlebotominae (Diptera: Psychodidae) of tropical dry forests in Northern Colombia. J Med Entomol 2002;39: 451-6.

58. Cárdenas R, Romo G, Santamaría E, Bello F, Ferro C. Lutzomyia longiflocosa (Diptera: Psychodidae) posible vector en el foco de leishmaniasis cutánea del municipio de Planadas, zona cafetera del Tolima. Biomédica 1999;19:239-44.

59. Sierra D, Vélez ID, Uribe S. Identificación de Lutzomyia spp. (Diptera: Psychodidae) grupo verrucarum por medio de microscopía electrónica de sus huevos. Rev Biol Trop 2000;48:615-22.

60. Morales A, Corredor A, Cáceres E, Ibagos AL, de Rodriguez C. Aislamiento de tres cepas de Leishmania a partir de Lutzomyia trapidoi en colombia. Biomédica 1981;1:198-206.

61. Alexander B, Jaramillo C, Usma MC, Quesada BL, Cadena H, Roa W, et al. An attempt to control phlebotomine sand flies (Diptera: Psychodidae) by residual spraying with deltamethrin in a Colombian village. Mem Inst Oswaldo Cruz1995;90:421-4.

62. Barreto P, Montoya J, Solarte Y, Jaramillo C, Barreto M, Freyre JL, et al. Notas sobre algunos flebótomos Lutzomyia (Diptera: Psychodidae) en tres focos de leishmaniasis cutánea en el occidente de Colombia. Colombia Med 1989;20:53-9.

63. Jaramillo C, Montoya-Lerma J. Insectos flebótomos (Diptera: Psychodidae) del Valle del Cauca. Memorias, I Simposio Nacional de Fauna del Valle del Cauca, Cali, Colombia; 1991. p.167-74.

64. Alexander B. Biology and control of sand flies in Colombian coffee plantations. Bol Dir Malariol y San Amb 1995;35:21-8.

65. Santamaría E, Munstermann LE, Ferro C. Estimating carrying capacity in a newly colonized sand fly Lutzomyia serrana (Diptera: Psychodidae). J Econ Entomol 2002;95:149-54.

66. Montoya J, Jaramillo C, Palma G, Gómez T, Segura I, Travi B. Report of an epidemic outbreak of tegumentary leishmaniasis in a coffee-growing area of Colombia. Mem Inst Oswaldo Cruz 1990;85:119-21.
67. Testa JM, Montoya-Lerma J, Cadena H, Oviedo M, Ready PD. Molecular identification of vectors of Leishmania in Colombia: mitochondrial introgression in the Lutzomyia townsendi series. Acta Trop 2002; 84: 205-18.

68. Cárdenas R, Gutiérrez M, Angulo VM, Munstermann L, Sandoval M, Ferro C. Phlebotomine sand flies (Diptera: Psychodidae) habitat associations to urban cutaneous leishmaniasis in the city of Bucaramanga, Northwestern Colombia. Abstracts, Vol. $2 \mathrm{XV}$ International Congress for Tropical Medicine and Malaria, Cartagena de Indias, Colombia; 2000. p.68.

69. Ferro C, Morales A. Flebótomos de Colombia: Estudios realizados por el Laboratorio de Entomología, 19651997. En: Toro G, Hernández CA, Raad J, editores. Instituto Nacional de Salud 1917-1997. Una historia, un compromiso. Santafé de Bogotá: INS; 1998. p.219-33.

70. Santamaría E, Castillo M, Cárdenas R, Bello F, Ayala M, Ferro C. Competencia vectorial de las especies de Lutzomyia del grupo verrucarum (Diptera, Psychodidae) en un foco endémico de Leishmania braziliensis en Reventones, Cundinamarca. Biomédica 1999;19:11526.

71. Santamaría E, Castillo M, Cárdenas R, Bello F, Ayala M, Ferro C. Transmisión experimental de Leishmania braziliensis a hámster por picadura de Lutzomyia longiflocosa (Diptera: Psychodidae) provenientes de un foco endémico en la zona cafetera colombiana. Médicas UIS 1998;12:279-84.

72. Travi BL, Ferro C, Cadena H, Montoya-Lerma J, Adler GH. Canine visceral leishmaniasis: dog infectivity to sand flies from non-endemic areas. Res Vet Sci 2002; 72:83-6.

73. Arroyo CG, Garzón J. Investigación de un foco de leishmaniasis cutánea en la zona andina del departamento de Nariño. Biomédica 1996;16:25-31.

74. Montoya-Lerma J, Cadena H, Segura I, Travi BL. Association of Lutzomyia columbiana (Diptera: Psychodidae) with a leishmaniasis focus in Colombia due to species of the Leishmania mexicana complex. Mem Inst Oswaldo Cruz 1999;94:277-83.

75. Alexander B, Usma MC, Cadena H, Quesada BL, Solarte Y, Roa W, et al. Phlebotomine sandflies associated with a focus of cutaneous leishmaniasis in Valle del Cauca, Colombia. Med Vet Entomol 1995; 9: 273-8.

76. Van der Hammen T. Aspectos de historia y ecología de la biodiversidad norandina y amazónica. Rev Acad Colomb Cienc 2000;24:231-45.

77. Bejarano EE. Nuevas herramientas para la clasificación taxonómica de los insectos vectores de leishmaniosis: utilidad de los genes mitocondriales. Biomédica $2001 ; 21: 182-91$ 